\title{
Implementation of novel processes in existing process networks: evaluation of the industrial production of $\mathrm{C}_{4}$ chemicals by optimization
}

\author{
Gerard P.J. Dijkema ${ }^{1}$, Ellis M. Steenkamp ${ }^{2, *}$ and Peter J.T. Verheijen ${ }^{2}$ \\ ${ }^{1}$ Dept. of Systems Engineering, Policy Analysis and Management \\ ${ }^{2}$ Dept. of Chemical Engineering, Delft University of Technology \\ Julianalaan 134, 2628 BL Delft, The Netherlands.
}

\begin{abstract}
An analysis method of optimal network flexibility was developed and implemented in MATLAB. The use of novel processes in the existing Dutch $\mathrm{C}_{4}$-network was assessed. Since its optimal structure is determined by only two processes, development of these must be given priority. The network has substantial flexibility with respect to total mass use, but almost none when total energy usage is considered. Some novel processe therefore may not be implemented if profits are largely determined by total cost of feedstock.
\end{abstract}

\section{INTRODUCTION}

The development of the chemical industry is driven by market demand, feedstock supply, production cost, and legislation. We have assumed that these forces push the chemical industry continuously towards a situation that is optimal within the prevailing technological, economic, and ecological constraints. As the chemical industry is a complex of processes, one way to improve its performance is to implement novel processes in existing process networks. Since the end of the sixties, however, the number of novel processes put into industrial practice per decade has decreased. This has been attributed to the maturation of the chemical industry and to the decline in its growth rate. In addition, the very existence of complex process networks in this industry may also play a role, since novel processes must not only fit into an existing network, they must also improve the total network's performance. What are the effects of this dual network requirement on the development of new processes and their use in industrial practice, and what lessons are to be learned for the management of process research and development?

Stadtherr and Rudd (1976, 1978a, 1978b) implemented a linear programming (LP) model of a total industry to examine changes in the structure of the American chemical processing industry from 1940 to 1970 . They calculated a selection of processes out of a set of available processes that expanded in 10-year intervals and compared the results with the actual developments in the industry. In essence, they concluded that the optimum of their LPmodel with respect to resource usage was an adequate approximation of the actual process network structure and its capacities. We applied this model therefore to the Dutch industrial process network for the production of $\mathrm{C}_{4}$ chemicals as it existed in 1990, in order to simulate the introduction of new processes (Cornelissen, 1995).

In this paper, we report the development of the LPmodel into a tool that could be employed in decision making. We summarize the mathematical formulation of the LP-problem and its extension that was required both to visualize network flexibility and to obtain reliable numerical results. Secondly, we describe the case study network that was modeled as a system of processes that are connected by mass and energy flows. Each process was modeled as a black box that is defined solely by its inputs and outputs. The available data had been used in several studies, as the network could be considered a relatively independent part of the industry. The third section covers the assessment of optimization objectives, which can be based on resource consumption, energy use and costs. Subsequently, we report on the model calculations that were done in MATLAB, as this is a widely available tool that includes flexible presentation capabilities and a compact language. We give the results of a number of numerical experiments. Finally, we derive from these results some criteria and heuristics on the development of $\mathrm{C}_{4}$ processes that may be used to focus both exploratory research into novel processes and research and development on process improvement.

\section{MATHEMATICAL FORMULATION}

The vector of variables, $\mathbf{x} \in \mathbb{R}^{n+2 m}$, includes those that must be determined by solving the optimization problem. In our model this vector consists of the capacity of each

\footnotetext{
*Present address: DSM, P.O. Box 606, Geleen, The Netherlands
} 
process, $c \in \mathbb{R}^{n}$, the network's resource or feed streams, $\mathbf{r} \in \mathbb{R}^{m}$, and the network product streams, $\mathbf{p} \in \mathbb{R}^{m}$, therefore

$$
\mathbf{x}=\left[\begin{array}{l}
\mathbf{c} \\
\mathbf{r} \\
\mathbf{p}
\end{array}\right]
$$

The conversion of feedstocks into products for the set of available processes has been modeled by the $m \times n$ matrix $A_{c}$, the technology matrix (Sophos et al., 1980), where $m$ represents the number substances in the network and $n$ the number of distinct processes. In $A_{c}$ element $a_{i j}$ is the input-output coefficient of substance $i=1 \ldots m$ for process $j=1 \ldots n$ and

$$
A_{c} \mathbf{c}+\mathbf{r}-\mathbf{p}=\mathbf{0} .
$$

All process input-output coefficients have been based on overall stoechiometry and are assumed to be constant. The equation above can be rewritten towards the standard LP form after $A_{c}$ has been expanded to yield the matrix A with

$$
A=\left[\begin{array}{lll}
A_{c} & I & -I
\end{array}\right]
$$

and $I$ the $m \times m$ identity matrix. A set of inequality relations models the market demand (d) as the lower limit on the appropriate network product streams, and the maximum feed stream supplies (s) as a set of upper limits. All variables $\mathbf{x}$ must be non-negative. The standard LP model is completed by an objective function that is a linear combination of these variables, $f^{T} x$. The coefficients in vector $\mathbf{f}$ determine the nature of the optimization; $f_{j}$ can represent carbon-in-feed use, energy use, exergy use, or economics per stream and process. The minimization problem is then

$$
\min _{\mathbf{x}} \mathbf{f}^{T} \mathbf{x}
$$

subject to

$$
\begin{aligned}
A \mathbf{x} & =\mathbf{0} \\
\mathbf{r} & \leq \mathbf{s}, \quad \mathbf{p} \geq \mathbf{d}, \quad \mathbf{x} \geq \mathbf{0}
\end{aligned}
$$

A solution to this optimization problem, $\mathbf{x}_{0}$, yields in particular a set of process capacities, $\mathbf{c}_{0}$. This set describes the process network structure completely; if a process capacity is set to zero it is not included. The solution of this problem is degenerate, i.e. rather than one unique solution there exists a finite set of solutions. This finite set can be described by a matrix $N$. The columns of this matrix are vectors that span the nullspace of $A$ combined with the matrix describing the set of active inequality constraints,

$$
\mathbf{x}=\mathbf{x}_{0}+N \alpha
$$

The elements of $\alpha$ can be choosen freely within the limitations set by the contraints of the optimization problem in Eq. (1). A row of zeroes in $N$ indicates that the capacity of the associated process cannot be varied, i.e. the process is either included in the network at a fixed capacity or it is not included. In the other case a range of non-negative values can be given for variables $x_{i}$, which implies that these variables have slack, which is a measure of flexibility. The minimum and maximum of a variable $x_{i}$ follows from respectively

and $\begin{array}{ll} & \min _{\mathbf{x}} x_{i} \\ & \max _{\mathbf{x}} x_{i}\end{array}$

both subject to

$$
\begin{aligned}
A \mathbf{x} & =\mathbf{0} \\
\mathbf{f}^{T} \mathbf{x} & =\mathbf{f}^{T} \mathbf{x}_{0} \\
\mathbf{r} & \leq \mathbf{s}, \quad \mathbf{p} \geq \mathbf{d}, \quad \mathbf{x} \geq \mathbf{0}
\end{aligned}
$$

Flexibility or slack can thus be determined by solving two new LP problems, subject to the constraints of the original problem and the extra condition that only solutions equal to the optimum of Eq. (1) should be maintained.

It proved useful to transform the vector $\mathbf{x}$ to the linear combinations specified in Eq. (2), because it reduces the dimension of the search space. So there is effectively a two-step approach to find flexibility. This approach also was useful in the investigation of multi-objective problems, where we looked for the optimum for a second objective that lies within the solution space of the optimum of the first objective.

An NLP problem resulted in case the economics of the network was modeled by an objective of the form

$$
\mathbf{f}_{c}^{T} \mathbf{c}^{\gamma}+\mathbf{f}_{r}^{T} \mathbf{r}+\mathbf{f}_{p}^{T} \mathbf{p}
$$

where $\gamma$ was choosen to be 0.6 ; and the constants, $\mathbf{f}_{c}$, and prices, $\mathbf{f}_{r}$ and $\mathbf{f}_{p}$, were estimated.

\section{CASE DESCRIPTION}

A process network was studied that has been based on 1990-data on the industrial production of $\mathrm{C}_{4}$-chemicals in the Netherlands (Chemical Intelligence Services, 1991). In addition, process data of sufficient detail could be taken from Chauvel and Lefebvre (1989) and Hydrocarbon Processing (1995). We have defined a base case of 18 processes, which presents the situation in 1990 . We present here a single extended case, which has another six processes added to it. Both cases are illustrated in Fig. 1.

The base case network uses two $\mathrm{C}_{4}$-rich feedstocks, the $\mathrm{C}_{4}$ byproduct of a steamcracker, which produces ethylene and propylene as primary products, and a butane-rich stream that either originates from a refinery as LPG or from natural gas winning as condensate. The network produces butadiene, MTBE, MEK, tert-butyl-alcolhol (TBA), and 1-butene. Propene oxide (PO) is a byproduct formed from propene in the TBA process. As shown, a number of different production routes can be applied to obtain the limited number of products that are commercially important.

In the past years a number of new industrial processes have become available to convert $\mathrm{C}_{4}$ 's and other feedstocks into these products. Processes have been developed and employed worldwide to cater for the rapidly growing markets for MTBE and 1-butene. MTBE is a 


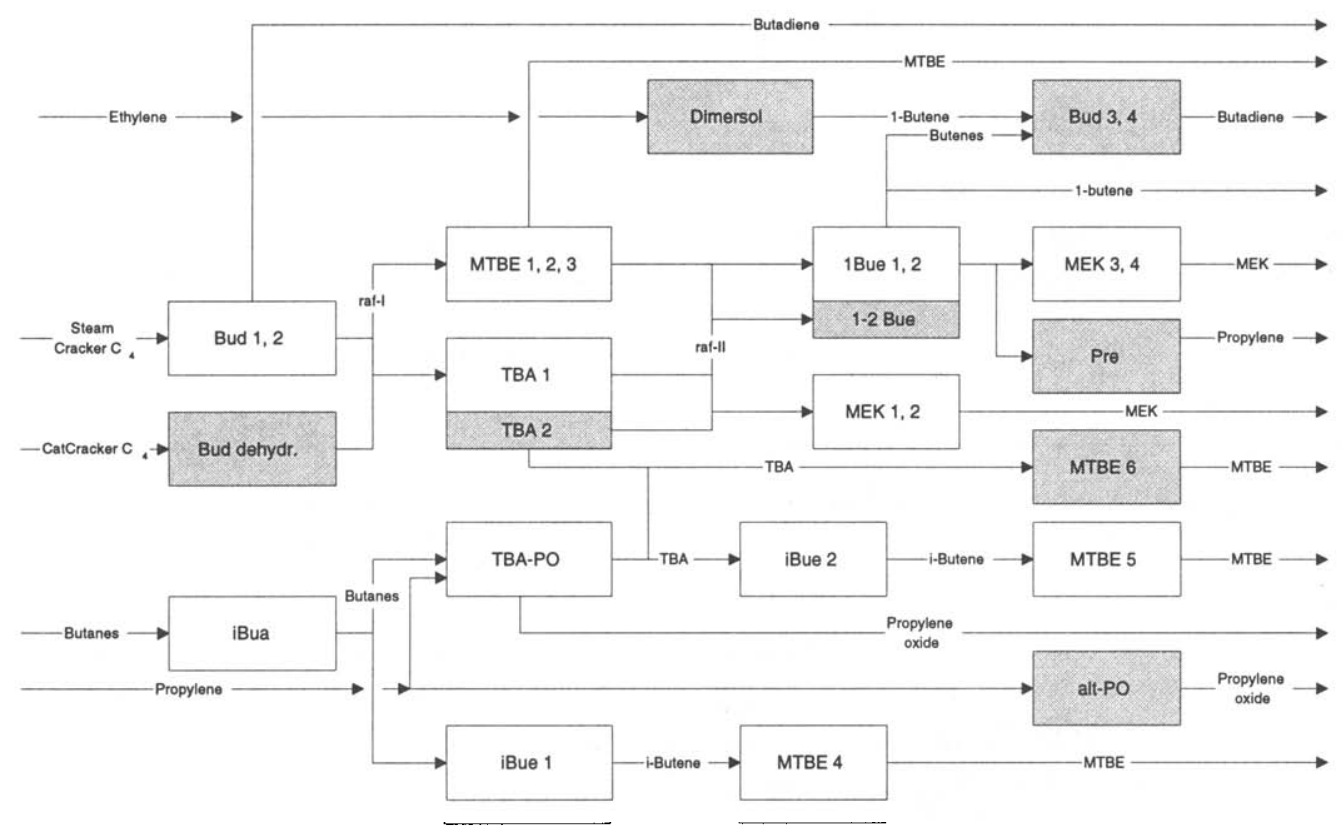

Figure 1: The 1990 network of $\mathrm{C}_{4}$ processes in The Netherlands. The unshaded processes constitute the base case. The shaded units are new processes included with the base case into the extended case.

gasoline octane-booster that replaces lead-compounds; 1 butene is an important component of LLDPE, linear-lowdensity polyethylene, which is a fast-growing bulk-plastic. Since butadiene extraction from $\mathrm{C}_{4}$-steamcracker cuts is the main source for butadiene worldwide, its supply is closely related to the production volume of ethylene, the primary product of a steamcracker. Alternative butadiene processes have been developed and additional butadiene outlets have been sought in order to make the butadiene market less dependent from ethylene, but none of these processes were used to date in industrial practice except for the dehydrogenation of butenes. In the extended case two additional feedstocks can be used. Ethylene can be dimerized to yield 1-butene and additional 1-butene can be obtained by extraction from catcracker $\mathrm{C}_{4}$ effluents. Propylene is added to the network products, as metathesis of 2-butene and ethene yields polymer grade propylene. A - still visionary - process for the direct oxidation of propylene to $\mathrm{PO}$ has been included to investigate the effects of the decoupling of TBA and PO production. The dehydrogenation of butenes was added, since these processes may be revived.

\section{OBJECTIVE FUNCTION}

The objective function for the network optimisation must model the forces that drive the operation of a network of chemical processes adequately. Although process designers must take into account a range of non-commensurable and conflicting objectives such as mass and energy efficiencies, reliability, safety, and operability (Sophos et al.,
1980), economics may be seen as the most important driving forces that shape the network structure. Market prices and investment costs therefore are the preferred data, however, these are not always easy to find or accessible. Moreover, these may have been largely determined by rapid market developments that influence short-range profitability only, whereas the profitability over a long time period prevails in the decision on the construction of new plants (Stadtherr and Rudd, 1976 and 1978b; Sokic and Stevancevic, 1983). In addition, Stadtherr and Rudd (1978b) observed that "apparently market prices are not true measures of a feedstock's value". They attribute this to internal deliveries amongst corporately integrated petroleum refiners and petrochemical producers that are not solely dictated by the open market. Therefore a model of the economic forces has been preferred that valuates the design variables in the optimisation problem, i.e. the process capacities and the process input-output relations. Since costof-feedstock accounts for $40-80 \%$ of production cost for most petrochemical processes and because carbon is the major backbone of the industries feedstock, Stadtherr and Rudd (1976) adopted carbon-in-feed minimization as a plausible alternative for economic optimization (Stadtherr and Rudd, 1978b). Sophos et al. (1980) included energy criteria in a multi-objective optimisation. As the combination of the lower heating value (LHV)of process streams and process utility use puts weights on both the streams and the processes in the network, this valuation model may be seen as a proper alternative to multi-objective optimization. In addition, the LHV is strongly correlated with both economics and the carbon content, the criterion used by Stadtherr and Rudd (1976), as the economic value is partly 
Table 1: Overview of objective functions for network optimization

\begin{tabular}{|c|c|c|}
\hline Objective & Description & $f$ in problem of Eq. (1) \\
\hline $\begin{array}{l}\text { MASS } \\
\text { total mass } \\
\text { carbon content }\end{array}$ & $\begin{array}{l}\Sigma \text { mass of network feed streams } \\
\Sigma \text { carbon content (network feed streams) }\end{array}$ & $\begin{array}{l}\text { unity for all feeds } \\
\text { carbon content for each feed }\end{array}$ \\
\hline \multicolumn{3}{|l|}{ ENERGY } \\
\hline Utility use & $\Sigma$ utility use (over all processes) & unity for all utilities \\
\hline Total energy use & $\begin{array}{l}\Sigma \text { utility use (processes) }+\Sigma \text { energy content (network } \\
\text { feed) }\end{array}$ & $\mathrm{LHV}[\mathrm{J} / \mathrm{kg}]$ for all streams \\
\hline \multirow[t]{2}{*}{ Useful energy } & $\begin{array}{l}\Sigma \text { utility use (processes) }+\Sigma \text { exergy content (network } \\
\text { feed) }\end{array}$ & electricity: 1 , steam: 0.5 , fuel: 0.8 \\
\hline & & and exergy value for all streams \\
\hline \multicolumn{3}{|l|}{$\mathrm{CO}_{2}$} \\
\hline Waste & waste is considered potential $\mathrm{CO}_{2}$ production & $\mathrm{CO}_{2}$ per unit waste $[\mathrm{kg} / \mathrm{kg}]$ \\
\hline $\mathrm{CO}_{2}$ utilities & $\mathrm{CO}_{2}$ production by utilities & $\mathrm{CO}_{2}$ per unit utility $[\mathrm{kg} / \mathrm{J}]$ \\
\hline \multicolumn{3}{|l|}{ ECONOMY } \\
\hline Input costs & feed and utility costs & cost per unit stream [ECU/kg] \\
\hline Output costs & waste processing cost and $\Sigma \mathrm{CO}_{2}$ penalties & cost per unit stream [ECU/kg] \\
\hline Investments (lin.) & $\Sigma$ Investments $\sim c$ & cost per unit capacity $[\mathrm{ECU} / \mathrm{kg}]$ \\
\hline Investments & $\Sigma$ Investments $\sim c^{0.6}$ & as in eq. 3 \\
\hline
\end{tabular}

based on fuel value.

All objectives mentioned in Table 1 have been investigated for the base case studied here. The first purpose was to find the objective that leads to an optimum structure closely resembling the actual network. Secondly, a choice needed to be made which optimization model could be most usefully used in predicting the response to the introduction of new processes. So all possibilities needed to be studied.

\section{IMPLEMENTATION}

Cornelissen (1995) implemented the LP-model for the base case in GAMS (Brooke et al., (1992)). This software environment, however, requires a certain learning curve and is not transparent for non-expert users. Therefore the model was subsequently programmed in MATLAB (Mathworks, 1992). As this environment offers a programming language, graphical functions, and a user interface that are easy to use, we expected that the numerical experiments such as the comparison of all valuation models could be implemented and executed more rapidly. In addition, MATLAB includes sufficient optimization routines and its results can be analyzed interactively. A simple menu-driven system was set-up in order to read in data, check them for internal consistency, and to perform the optimization operation. We had to take a number of precautions, however, to ensure optimality of each optimum determined, because the use of multiple start conditions proved that convergence occurs sometimes at non-optimal points in case MATLAB's standard LP-solver is used. Secondly, when the solution is degenerate, MATLAB calculates a final, single solution as least squares sum over the constraints and no warning is issued. Both problems have been addressed by an analysis of the solution space around the optimum. A further optimization was carried out within this subspace in case its dimen- sion did not equal zero, which is an indication of the occurrence of a degenerate solution. An optimization run of the $\mathrm{C}_{4}$ network on average required an hour of computation when a $100 \mathrm{MHz}$ Pentium-PC was used. The verification of optimality consumed $30 \%$ of this time. The $\mathrm{C}_{4}$ network was modeled by about 100 variables and 130 equations for the constraints. The capacity slack was determined for all processes that could be selected for inclusion in the optimal network. As stated, economic optimization is an NLP problem, since the relation between investment cost and process capacities must be modeled by a simple power law. Since the computations required one day or more when using a Pentium-PC, the results were not easily amenable to further experimentation.

\section{RESULTS}

By optimization using total mass and carbon content as objective, Cornelissen (1995) found degenerate solutions that were explained in part by the shape of the objective function that was very flat around the optimum capacity of most processes. The variation of $\mathrm{PO}$ and butadiene process capacities largely effects the value of the objective function. The market demand for $\mathrm{PO}$ and butadiene therefore largely determine the optimal network structure, which may be understood by inspection of Fig. 1. The $\mathrm{PO}$ and butadiene demand favor the selection of processes that use butadiene and PO co-products as a feedstock over those that use alternative feedstocks. For the management of $R$ \& D this implies that the development of improvements or alternatives for these two processes must be given priority, since development of most other processes in the network will problaby not result in industrial use. The mass optimization was repeated in MATLAB and the flexibility in the network analysed. As we expected, out of a 
set of 18 processes 14 processes are included in the problem's solutionspace that can vary within a substantial capacity range.

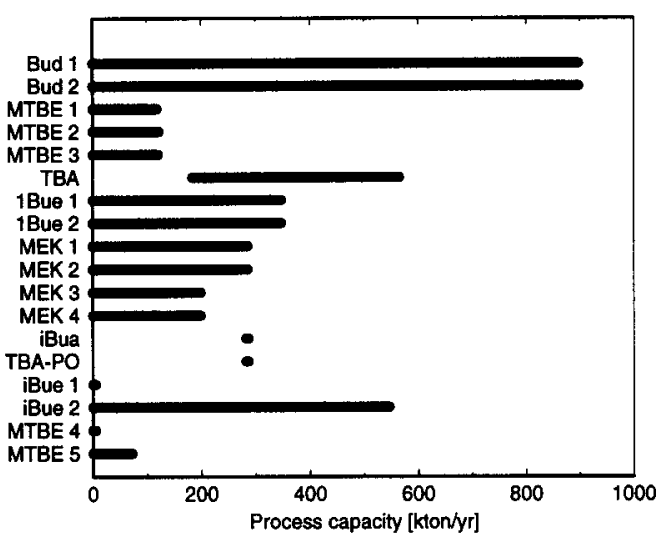

Figure 2: The range of flexibilities in the base case. In Fig. 2 we see that butadiene capacities can vary between the two available processes to meet the demand. The same is true for MTBE and TBA, which interchange for PO production. The slack in all other capacities is smaller because the network structure has largely been fixed by PO and butadiene processing capacity. Almost no degeneracy is present in the base case optimal solution when total energy was used. Apparently there exists a large amount of flexibility in the base case network solution if its economics are largely determined by feedstock cost, whereas the optimal solution becomes almost unique in case the cost of utilities are also accounted for.

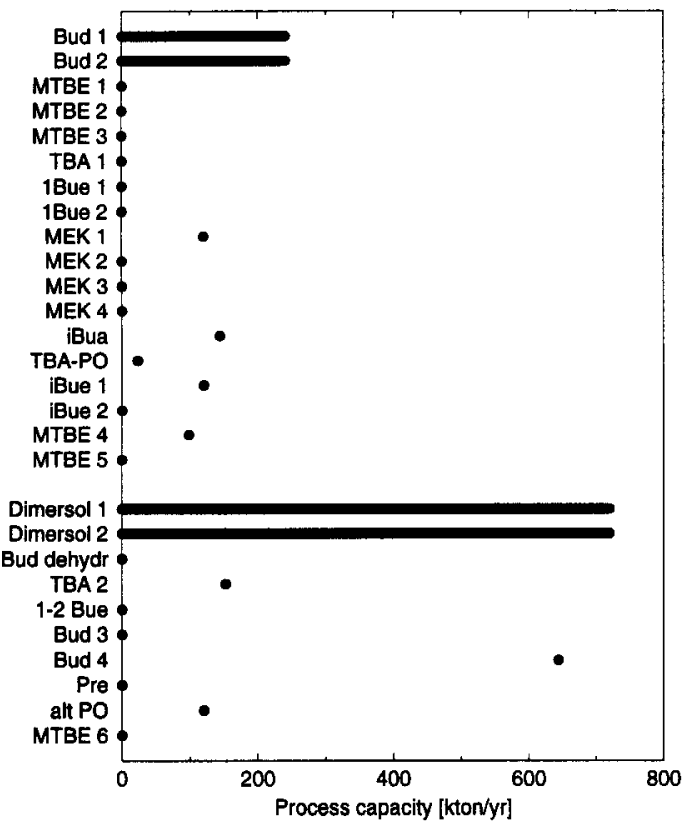

Figure 3: The range of flexibilities in the extended case. The availability of novel butadiene processes has a substantial effect on the network flexibility (Fig. 3). Although the capacity range of the butadiene processes is still large in the extended case, the network flexibility has decreased, as the capacity of 6 processes has become a fixed value. A change in the network structure that involves a novel process, therefore, may be optimal but may never be implemented since the optimal solution may be within the flexibility region of both base cäse and extended case.

\section{CONCLUSIONS}

We demonstrated that the industrial $\mathrm{C}_{4}$ process network has flexibility with respect to optimal use of feedstock, and that a limited number of processes largely determine its structure. This implies that the development of these processes must be given priority. The network flexibility reduces when the cost of utilities increases, which confirms that the actual use of new, energy-efficient processes is largely dependent on energy prices.

\section{NOMENCLATURE}

$A_{c}=$ technology matrix $(m \times n)$

$A=$ expanded technology matrix $(m \times(n+$ $2 m)$ )

$\mathbf{c}=$ vector of process capacities

$\mathbf{d}=$ vector of demand constraints

$\mathbf{f}=$ vector of objective function coefficients

$i=$ index

$m=$ the number of substances

$n=$ the number of processes

$N=$ see Eq. 2

$\mathbf{p}=$ vector of productstreams

$\mathbf{r}=$ vector of feedstreams

$\mathbf{s}=$ vector of supply constraints

$\mathbf{x}=$ vector that combines $\mathbf{c}, \mathbf{r}$ and $\mathbf{p}$

$\mathbf{x}_{0}=$ solution of the optimization problem

$\alpha=$ see Eq. 2

$\gamma=$ power relation investment vs capacity

\section{REFERENCES}

Chemical Intelligence Services, 1991, ChemFacts Netherlands, 1990, ICP Press, London.

Brooke, A., Kendrick, D., and Meeraus. A, 1992, GAMS, a User's Guide, The Scientific Press, San Francisco.

Chauvel, A., and Lefevbre G., 1989, Petrochemical Processes, Gulf. Publ., Houston.

Cornelissen, B.I., 1995 , The influence of technology development on a network of processes, Unpublished MSc Thesis, Delft University of Technology.

Mathworks, 1992, The MATLAB User's guide, The Mathworks Inc., Natick, MA, USA. 
Sokic, M. and Stevancevic, D, 1983, The optimal structure of the system of the petrochemical industry, Chem. Eng. Sci., 38, 265-273.

Sophos, A., Rotstein, E., and Stephanopoulos, G., 1980, Multiobjective analysis in modelling the petrochemical industry, Chem. Eng. Sci., 35, 2415-2426.
Stadtherr, M.A. and Rudd, D.F., 1976, Systems study of the petrochemical industry, Chem. Eng. Sci., 31, 1019 Stadtherr, M.A. and Rudd, D.F., 1978a, A systems approach to assessing new petrochemical industry, Chem. Eng. Sci., 33, 921

Stadtherr, M.A. and Rudd, D.F., 1978b, Resource use by the chemical industry, Chem. Eng. Sci., 33, 923 